\title{
Nonlinear instability of periodic BGK waves for Vlasov-Poisson system
}

\author{
Zhiwu Lin \\ Courant Institute
}

\begin{abstract}
We investigate the nonlinear instability of periodic Bernstein-Greene-Kruskal(BGK) waves. Starting from an exponentially growing mode to the linearized equation, we proved nonlinear instability in the $L^{1}$-norm of the electric field.
\end{abstract}

\section{Introduction}

We consider a one-dimensional electron plasma with a fixed homogeneous neutralizing ion background. In such a plasma collisions are relatively rare; hence we assume no collisions at all. So the time evolution can be modelled by the Vlasov-Poisson system

$$
\frac{\partial f}{\partial t}+v \frac{\partial f}{\partial x}-E \frac{\partial f}{\partial v}=0
$$

$$
\frac{\partial E}{\partial x}=-\int_{-\infty}^{+\infty} f d v+1
$$

where $f(x, v, t)$ is the electron distribution function, $E(x, t)=-\partial_{x} \phi(x, t)$ the electric field ( $\phi$ is the electric potential), and 1 is the ion density. In 1957, Bernstein, Greene and Kruskal ([2]) showed the existence of an infinite family of exact stationary solutions to (1), called BGK waves. Since then, the stability of these BGK waves has been of great interest. In [4], [5], [6], Guo and Strauss initiated a rigorous study of instability of spatially-dependent equilibria in plasmas. Using some delicate perturbation arguments, they proved in particular that inhomogeneous periodic BGK waves with small amplitude are linearly and nonlinearly unstable, if the corresponding homogeneous case has a growing mode according to Penrose's criteria. In [7], we show that an arbitrary periodic BGK wave is linearly unstable under multi-periodic perturbations. In this paper, we finally complete such instability investigation of BGK waves by showing that linear instability always implies nonlinear instability, without the smallness assumption. In particular, combining with the result in [7], we 
prove that any periodic BGK wave is nonlinearly unstable under multiperiodic perturbations. Before stating our main theorem, we introduce some notations as in [7].

A BGK wave is a steady state of the form

$$
f_{0}(x, v)=\mu\left(\frac{1}{2} v^{2}-\beta(x)\right), E_{0}(x)=-\beta_{x}(x),
$$

with $\beta$ a periodic solution of the differential equation

$$
\beta_{x x}=\int_{-\infty}^{+\infty} \mu\left(\frac{1}{2} v^{2}-\beta(x)\right) d v-1 .
$$

We assume that (i) $\mu(\theta)$ is a nonnegative $C^{2}$ function on $\mathbf{R}$; (ii) $\mu$ is neutral, that is,

$$
\int_{-\infty}^{+\infty} \mu\left(\frac{1}{2} v^{2}\right) d v-1=0
$$

and (iii) For some $\gamma>1$

$$
\left|\mu^{\prime}(\theta)\right|,\left|\mu^{\prime \prime}(\theta)\right| \leq \frac{C}{1+|\theta|^{\gamma}}
$$

Let $\beta$ be any periodic solution of (1.2) and $P_{\beta}$ be its minimal period. By adjusting the starting point, we can arrange that the solution satisfies:

$$
\begin{gathered}
\beta(0)=\beta\left(P_{\beta}\right)=\min _{0 \leq x \leq P_{\beta}} \beta(x), \quad \beta\left(\frac{P_{\beta}}{2}\right)=\max _{0 \leq x \leq P_{\beta}} \beta(x), \\
\beta(x)=\beta\left(P_{\beta}-x\right), \quad \forall x \in\left[0, P_{\beta}\right],
\end{gathered}
$$

and $\beta(x)$ is strictly increasing in $\left[0, \frac{P_{\beta}}{2}\right]$. Notice that by extending $\beta(x)$ to $\left[0, k P_{\beta}\right]$ periodically, we obtain new BGK waves with period $k P_{\beta}$. It was proved in [7] that if $k \geq 2$, then there exists some $k P_{\beta}$-periodic growing mode to the linearized equation. That is, there is a solution $\left(e^{\lambda t} \tilde{f}, e^{\lambda t} \tilde{E}\right)$ with $\lambda>0$ to

$$
\begin{gathered}
\frac{\partial f}{\partial t}+v \frac{\partial f}{\partial x}-E_{0} \frac{\partial f}{\partial v}=E \frac{\partial f_{0}}{\partial v}, \\
\frac{\partial E}{\partial x}=-\int_{-\infty}^{+\infty} f d v
\end{gathered}
$$

where $\tilde{f}$ and $\tilde{E}$ are $k P_{\beta}$-periodic functions in $x$ and $\tilde{E} \in H^{1}$. The main theorem in this paper is 
TheOREm 1.1 Let $\left(E_{0}, f_{0}\right)$ be the above periodic BGK solution to (1) with period $P=k P_{\beta}(k \geq 2)$ and $\mu$ satisfying (1.4) and (3.1). Then there exists positive constants $\varepsilon_{0}, C_{1}$ and a family of solutions

$$
\left[\bar{f}^{\delta}(t), \bar{E}^{\delta}(t)\right] \in W^{1,1}([0, P] \times \mathbf{R})
$$

of (1) with period $P$ in $x$, defined for $\delta$ sufficiently small, with $\bar{f}^{\delta}(t)$ non-negative, such that

$$
\left\|\bar{f}^{\delta}(0)-\mu\left(\frac{1}{2} v^{2}-\beta(x)\right)\right\|_{W^{1,1}([0, P] \times \mathbf{R})}+\left\|\bar{E}^{\delta}(0)-E_{0}\right\|_{W^{1,1}(0, P)} \leq \delta,
$$

and

$$
\sup _{0 \leq t \leq C_{1}|\ln \delta|}\left\|\bar{E}^{\delta}(t)-E_{0}\right\|_{L^{1}(0, P)} \geq \varepsilon_{0} .
$$

We note that in [4] [5], nonlinear instability is proved in terms of the $\mathbf{L}^{1}$ norm of the electric field plus the electron distribution function. In our result, nonlinear instability is in the $\mathbf{L}^{1}$ norm of the electric field only, which is clearer physically. In particular, this implies that the deviation from the unstable BGK waves is macroscopic, not in the microscopic sense.

Before sketching the main idea of proving Theorem 1.1, we indicate some difficulties in the nonlinear instability study. The main difficulty is that the nonlinear term of (1), $E \partial_{v} f$, contains derivative and thus is unbounded in $\mathbf{L}^{p}$ space of $E$ and $f$. This is a typical situation in many equations in continuum physics and kinetic theory, and so far there is no general method to dealt with it. Starting with [4], Guo and Strauss developed a bootstrap technique to overcome this difficulty and prove nonlinear instability. Their basis idea is to derive the growth estimate for the derivative of the perturbation from the growth of the perturbation, in a time period during which the perturbation is exponentially growing while the amplitude is kept small. In [4] [5], they used this technique to bootstrap $\partial_{v} f$ form the estimate of $f$, under the assumption that the amplitude of the BGK wave is small. In [1], this technique was used to study nonlinear instability of ideal plane flows, with some new arguments. It was showed that if the growth rate of the growing mode exceeds the Liapunov exponent of the steady velocity field, then linear instability implies nonlinear instability. If we use the argument of [1] to the current problem, then similar result can be obtained. That is, nonlinear instabil- 
ity can only be showed if the growth rate exceeds the Liapunov exponent $\left(\sqrt{\beta_{x x}(0)}\right.$ in this case) of the particle equation.

To overcome such a difficulty, we introduce some new ideas. Let $E(t)$ and $f(t)$ be the small perturbations satisfying the nonlinear VlasovPoisson system (3). Our method is to study the $\mathbf{L}^{1}$ norm of $E(t)$ by using the following evolution formula for $f(t)$

$$
f(t)=e^{t L_{0}} f(0)+\int_{0}^{t} e^{(t-s) L_{0}} \partial_{v}(E f)(s) d s,
$$

where $L_{0}$ is the linearized operator. The idea of this coupled approach is to use the regularizing effect of Poisson's equation to get rid of the derivative $\partial_{v}$ in the nonlinear term $\partial_{v}(E f)$. In this way, the nonlinear term essentially becomes $E f$ which is easier to handle. To make this thinking formal, we use the following inequality to estimate $\|E(t)\|_{1}$

$$
\|E(t)\|_{1} \leq 2 \sup _{\substack{a \in \mathbf{W}^{1,1}(0, P) \\\left\|a_{x}\right\|_{\infty} \leq 1}} \iint_{[0, P] \times \mathbf{R}} f(t, x, v) a(x) d x d v
$$

and then use a duality argument and integration by parts to move $\partial_{v}$ in (1.10) to a differentiable test function. Another important point in our proof is that even though the quantity

$$
\frac{\partial \mathbf{X}(s ; x, v)}{\partial v}
$$

has pointwise exponential growth, where $(\mathbf{X}(s ; x, v), \mathbf{V}(s ; x, v))$ satisfies the particle trajectory equation (2), the integral

$$
\int\left|\frac{\partial \mathbf{X}(s ; x, v)}{\partial v}\right| d v
$$

only has linear growth. This is due to the geometric property of the particle trajectory. This observation is crucial in our proof of regularity of growing modes and nonlinear instability.

The method of proving Theorem 1.1 can be directly used to show that linear instability implies nonlinear instability of any periodic BGK waves, in the case of two species of particles or relativistic case. Moreover the new ideas we introduced in this paper are quite flexible and could apply to other physical systems. In ([9]), we use these new ideas to study general 2D ideal flows and prove nonlinear instability from linear instability, without assumption on the linear growth rate. 


\section{Regularity of growing modes}

In the following, we denote $P=k P_{\beta}$. First, we recall some facts about growing modes proved in [7]. The growing mode found in [7] is of the form $\left(e^{\lambda t} f, e^{\lambda t} E\right)$ with $\lambda>0, E=-\partial_{x} \phi$ and

$$
f(x, v)=-\mu^{\prime}(e) \phi(x)+\mu^{\prime}(e) \int_{-\infty}^{0} \lambda e^{\lambda s} \phi(\mathbf{X}(s ; x, v)) d s .
$$

Here $\phi \in \mathbf{H}_{\text {per }}^{2}(0, P)$ satisfies

$$
-\frac{\partial^{2}}{\partial x^{2}} \phi-\int_{\mathbf{R}} \mu^{\prime}(e) d v \phi(x)+\int_{\mathbf{R}} \mu^{\prime}(e) \int_{-\infty}^{0} \lambda e^{\lambda s} \phi(\mathbf{X}(s)) d s d v=0
$$

where $e=\frac{1}{2} v^{2}-\beta(x)$, and $(\mathbf{X}(s ; x, v), \mathbf{V}(s ; x, v))$ is the solution of the characteristic equation

$$
\begin{gathered}
\dot{\mathbf{X}}(s)=\mathbf{V}(s), \\
\dot{\mathbf{V}}(s)=-E_{0}(\mathbf{X}(s)),
\end{gathered}
$$

with initial data $\mathbf{X}(0)=x, \mathbf{V}(0)=v$. It was showed in [8] that the growing mode

$$
\left(e^{\lambda t} f, e^{\lambda t} E\right)
$$

satisfies (1) weakly and $f$ is almost everywhere differentiable in $x, v$. Now we show that

Theorem 2.1 The function $f$ defined by (2.1) is in $\mathbf{W}^{1,1}([0, P] \times \mathbf{R})$.

To see the main difficulty in the proof, we take the $v$-derivative of (2.1) and the following term appears in the right hand side

$$
I(x, v):=\mu^{\prime}(e) \int_{-\infty}^{0} \lambda e^{\lambda s} \phi_{x}(\mathbf{X}(s)) \frac{\partial \mathbf{X}(s)}{\partial v} d s .
$$

If the particle energy $e=-\beta_{\text {min }}$, then $\frac{\partial \mathbf{X}(s)}{\partial x}$ grows exponentially in $|s|$ with the rate $\sqrt{\beta_{x x}(0)}$. Thus if $\lambda<\sqrt{\beta_{x x}(0)}$, the integral in (2.3) might diverge and $I(x, v)$ becomes infinite on the critical set

$$
A_{\mathrm{cr}}=\left\{(x, v) \mid \frac{1}{2} v^{2}-\beta(x)=-\beta_{\min }\right\} .
$$

However we can show that $I(x, v) \in \mathbf{L}^{1}$. This is the following lemma. 
Lemma 2.2 The function $I(x, v)$ defined by (2.3) is in $\mathbf{L}^{1}([0, P] \times \mathbf{R})$.

Proof: We need to show that

$$
\int_{[0, P]} \int_{\mathbf{R}} \int_{-\infty}^{0} e^{\lambda s}\left|\mu^{\prime}(e) \phi_{x}(\mathbf{X}(s)) \frac{\partial \mathbf{X}(s)}{\partial v}\right| d s d v d x<\infty .
$$

Let $e_{\mathrm{cr}}=-\beta_{\min }$ and $e_{0}<e_{\mathrm{cr}}<e_{1}<e_{2}$ be three numbers to be determined later. We divide the $(x, v)$ space into the following five regions.

$\left(D_{1}\right)$ (fast particles) $e=\frac{1}{2} v^{2}-\beta(x)>e_{2}$.

$\left(D_{2}\right)$ (mildly free particles) $e_{1}<e<e_{2}$.

$\left(D_{3}\right)$ (barely free particles) $e_{\mathrm{cr}}<e<e_{1}$.

$\left(D_{4}\right)$ (barely trapped particles) $e_{0}<e<e_{\mathrm{cr}}$.

$\left(D_{5}\right)$ (strongly trapped particles) $-\beta_{\max } \leq e<e_{0}$. First we claim:

For $e_{2}$ sufficiently large, if $(x, v) \in D_{1}$ then there exists constant $C_{1}$ such that

$$
\left|\frac{\partial \mathbf{X}(s ; x, v)}{\partial v}\right| \leq C_{1} e^{\frac{\lambda}{2}|s|}
$$

for $-\infty \leq s \leq+\infty$.

To show (2.5), we consider the case of positive time and positive velocity only since (2) is time reversible. Differentiating (2.2a) with respect to $v$, we get

$$
\frac{\partial \dot{\mathbf{X}}(s)}{\partial v}=\frac{\partial \mathbf{V}(s)}{\partial v}
$$

So

$$
\frac{\partial \mathbf{X}(s)}{\partial v}=\int_{0}^{s} \frac{\partial \mathbf{V}(u)}{\partial v} d u
$$

For any solution $((\mathbf{X}(s ; x, v), \mathbf{V}(s ; x, v)))$ of $(2)$, we have

$$
\frac{1}{2} v^{2}-\beta(x)=\frac{1}{2} \mathbf{V}(s)^{2}-\beta(\mathbf{X}(s)) .
$$

Differentiating above with respect to $v$, we get

$$
v=\mathbf{V}(s) \frac{\partial \mathbf{V}(s)}{\partial v}-\beta_{x}(\mathbf{X}(s)) \frac{\partial \mathbf{X}(s)}{\partial v}
$$

It follows from (2.6) and (2.8) that

$$
\frac{\partial \mathbf{X}(s)}{\partial v}=\int_{0}^{s}\left(\frac{v}{\mathbf{V}(u)}+\frac{\beta_{x}(\mathbf{X}(u))}{\mathbf{V}(u)} \frac{\partial \mathbf{X}(u)}{\partial v}\right) d u .
$$


It is easy to see that if $e_{2}$ is large and $(x, v) \in D_{1}$, then we have

$$
\frac{1}{2} \leq \frac{v}{\mathbf{V}(u)} \leq \frac{3}{2},\left|\frac{\beta_{x}(\mathbf{X}(u))}{\mathbf{V}(u)}\right|<\frac{\lambda}{2}
$$

for $0 \leq u \leq+\infty$. Thus we have

$$
\frac{\partial \mathbf{X}(s)}{\partial v} \leq \frac{3}{2} s+\frac{\lambda}{2} \int_{0}^{S} \frac{\partial \mathbf{X}(u)}{\partial v} d u
$$

and the estimate (2.5) follows from Gronwall's inequality.

Since $D_{2}$ and $D_{5}$ are bounded and the Liapunov exponent of (2) is zero there, there exists some constant $C_{2}$ such that

$$
\left|\frac{\partial \mathbf{X}(s)}{\partial v}\right| \leq C_{2} e^{\frac{\lambda}{2}|s|},
$$

for $-\infty \leq s \leq+\infty$. Let $C_{3}=\max \left\{C_{1}, C_{2}\right\}$, then

$$
\begin{aligned}
& \iint_{D_{1} \cup D_{2} \cup D_{5}} \int_{-\infty}^{0} e^{\lambda s}\left|\mu^{\prime}(e) \phi_{x}(\mathbf{X}(s)) \frac{\partial \mathbf{X}(s)}{\partial v}\right| d s d v d x \\
\leq & \int_{-\infty}^{0} C_{3} e^{\frac{\lambda}{2} s} \iint_{D_{1} \cup D_{2} \cup D_{5}}\left|\mu^{\prime}(e) \phi_{x}(\mathbf{X}(s))\right| d v d x d s \\
\leq & \int_{-\infty}^{0} C_{3} e^{\frac{\lambda}{2} s} \int_{[0, P]} \int_{\mathbf{R}}\left|\mu^{\prime}(e) \phi_{x}(x)\right| d v d x \\
\leq & \int_{-\infty}^{0} C_{3} e^{\frac{\lambda}{2} s} \sup _{x} \int_{\mathbf{R}}\left|\mu^{\prime}(e)\right| d v \int_{[0, P]}\left|\phi_{x}(x)\right| d x \\
\leq & C^{\prime}\left(\int_{[0, P]}\left|\phi_{x}(x)\right|^{2} d x\right)^{\frac{1}{2}},
\end{aligned}
$$

which implies that

$$
\iint_{D_{1} \cup D_{2} \cup D_{5}}|I(x, v)| d x d v<\infty .
$$

Here in the second inequality above, we used the fact that the mapping

$$
(x, v) \rightarrow((\mathbf{X}(s ; x, v), \mathbf{V}(s ; x, v)))
$$

is $1-1$ with Jacobian 1

In $D_{3}$ and $D_{4}, \frac{\partial \mathbf{X}(s)}{\partial v}$ might have growth $e^{\sqrt{\beta_{x x}(0)} s}$ and we need to do more delicate analysis. First we recall some basic facts about the particle 
motion satisfying (2). If $e \neq e_{\mathrm{cr}}$, particles with energy $e$ do periodic motion. Free particles with energy $e>e_{\mathrm{cr}}$ travel through the whole interval $[0, P]$ with the period

$$
T(e)=\int_{0}^{P} \frac{1}{\sqrt{2(e+\beta(y))}} d y .
$$

and trapped particles with energy $e<e_{\mathrm{cr}}$ go back and forth within one of the intervals

$$
[a(e), b(e)],\left[a+P_{\beta}, b+P_{\beta}\right], \cdots,\left[a+(k-1) P_{\beta}, b+(k-1) P_{\beta}\right]
$$

with period

$$
T(e)=2 \int_{a(e)}^{b(e)} \frac{1}{\sqrt{2(e+\beta(y))}} d y .
$$

Here $a(e), b(e)$ are two points in $\left[0, P_{\beta}\right]$ such that $\beta(x)=-e$. For particles with energy $e_{\mathrm{cr}}$, it takes infinite time to approach the saddle point. We have the following properties of $T(e)$ :

(I) $T(e)$ is twice differentiable for $\left(-\beta_{\max }, e_{\mathrm{cr}}\right) \cup\left(e_{\mathrm{cr}}, \infty\right)$.

(II) $T(e) \rightarrow+\infty$ as $e \rightarrow e_{\mathrm{cr}}$ and $T(e) \rightarrow \frac{2 \pi}{\sqrt{\beta_{x x}(0)}}$ as $e \rightarrow-\beta_{\max }$.

(III) $T^{\prime}(e)<0$ for $e>e_{\mathrm{cr}}$

(IV) There exists $e_{0} \in\left(-\beta_{\max }, e_{\mathrm{cr}}\right)$ such that $T^{\prime}(e), T^{\prime \prime}(e)>0$ for $e_{0}<e<e_{\mathrm{cr}}$.

Properties (I),(II) are standard (e.g. see [3]) and (III) is obvious. It follows from (I) and (II) that $T^{\prime}(e), T^{\prime \prime}(e)$ tends to $+\infty$ as $e \rightarrow e_{\mathrm{cr}}+$. So if we choose $e_{0}$ close to $e_{\mathrm{cr}}$ then (IV) holds.

Now we estimate $\iint_{D_{3}}|I(x, v)| d x d v$. Let $J_{x}=\left\{v \mid(x, v) \in D_{3}\right\}, J_{x}^{+}=$ $J_{x} \cap\{v>0\}$ and $J_{x}^{-}=J_{x} \cap\{v<0\}$. Then

$$
\begin{aligned}
& \iint_{D_{3}}|I(x, v)| d x d v \\
& =\int_{-\infty}^{0} e^{\lambda s} \int_{[0, P]} \int_{J_{x}^{+} \cup J_{x}^{-}}\left|\mu^{\prime}(e) \phi_{x}(\mathbf{X}(s)) \frac{\partial \mathbf{X}(s)}{\partial v}\right| d v d x d s \\
& \leq C^{\prime \prime} \int_{-\infty}^{0} e^{\lambda s} \int_{[0, P]} \int_{J_{x}^{+} \cup J_{x}^{-}}\left|\frac{\partial \mathbf{X}(s)}{\partial v}\right| d v d x d s
\end{aligned}
$$

where

$$
C^{\prime \prime}=\left\|\mu^{\prime}(e)\right\|_{\infty}\left\|\phi_{x}\right\|_{\infty}
$$

We will prove that

$$
\int_{J_{x}^{+} \cup J_{x}^{-}}\left|\frac{\partial \mathbf{X}(s)}{\partial v}\right| d v \leq 2\left(\frac{|s|}{T\left(e_{1}\right)}+1\right) P .
$$


Then it follows from (2.10) and (2.11) that

$$
\iint_{D_{3}}|I(x, v)| d x d v<\infty .
$$

We only show (2.11) for positive $s$. First we notice that

$$
\int_{J_{x}^{+}}\left|\frac{\partial \mathbf{X}(s)}{\partial v}\right| d v=\int_{J_{x}^{+}} \frac{\partial \mathbf{X}(s)}{\partial v} d v=\int_{X \in \mathbf{X}\left(s ; J_{x}^{+}\right)} d X .
$$

Here in the second equality, we use the change of variable $v \rightarrow \mathbf{X}(s)$ and denote $\mathbf{X}\left(s ; J_{x}^{+}\right)$to be the image of $J_{x}^{+}$under this mapping. Free particle with energy $e>e_{\mathrm{cr}}$ always go in one direction. If they start from the same position $x$ with different velocity, they will never meet. The particles in $D_{3}$ have the minimal period $T\left(e_{1}\right)$. So by time $s$ they can finish at most $\left[\frac{s}{T\left(e_{1}\right)}\right]$ periods of motion and we can use $\left(\frac{s}{T\left(e_{1}\right)}+1\right)$ number of interval $[0, P]$ to cover the set $\mathbf{X}\left(s ; J_{x}^{+}\right)$. So

$$
\int_{J_{x}^{+}}\left|\frac{\partial \mathbf{X}(s)}{\partial v}\right| d v \leq\left(\frac{s}{T\left(e_{1}\right)}+1\right) P .
$$

The estimate on $J_{x}^{-}$is the same as above. Thus (2.11) is proved.

We use the same idea as above to estimate $\iint_{D_{4}}|I(x, v)| d x d v$. But particles in $D_{4}$ can go back and forth, we need to do more careful analysis. We use the same notations as above. We will prove that

$$
\int_{J_{x}^{+} \cup J_{x}^{-}}\left|\frac{\partial \mathbf{X}(s)}{\partial v}\right| d v \leq 4\left(\frac{|s|}{T\left(e_{0}\right)}+1\right) P_{\beta} .
$$

Assuming (2.13), then as what we did for $D_{3}$ above, we have

$$
\iint_{D_{4}}|I(x, v)| d x d v<\infty
$$

Combining with (2.9) and (2.12), we have

$$
I(x, v) \in \mathbf{L}^{1}([0, P] \times \mathbf{R}) .
$$

We only prove (2.13) for positive $s$ and $x \in\left(0, P_{\beta}\right)$ since the proof is the same for other cases. First by co-area formula, we have

$$
\int_{J_{x}^{+}}\left|\frac{\partial \mathbf{X}(s)}{\partial v}\right| d v=\int_{X \in \mathbf{X}\left(s ; J_{x}^{+}\right)} d X .
$$


So to show (2.13) we need to understand the set $\mathbf{X}\left(s ; J_{x}^{+}\right)$. We know that by time $s$, each particle in $D_{4}$ can finish at most $\left[\frac{s}{T\left(e_{0}\right)}\right]$ periods of motion. Consider the following question: for particles starting from the same position $x$ and with different energy $e \in\left(e_{0}, e_{\mathrm{cr}}\right)$, how many of them can collide at $x_{1}$ by time $s$ ? We classify the possible collision in the following way. Denote $P_{j}^{+}\left(P_{j}^{+}\right)$to be the set of all particles getting to $x_{1}$ at time $s$ with positive (negative) velocity after having exactly finished $j$ periods of motion and $n_{j}^{+}\left(n_{j}^{-}\right)$is the number of particles in $P_{j}^{+}\left(P_{j}^{+}\right)$. Note that as mentioned above, we have $0 \leq j \leq\left[\frac{s}{T\left(e_{0}\right)}\right]$. For a particle in $P_{j}^{+}$with energy $e$, we have

$$
s=j T(e)+\int_{x_{0}}^{x_{1}} \frac{d x}{\sqrt{2(e+\beta(x))}}
$$

if $x_{1}>x_{0}$ and

$$
s=j T(e)+T(e)-\int_{x_{1}}^{x_{0}} \frac{d x}{\sqrt{2(e+\beta(x))}}
$$

if $x_{1}<x_{0}$. For a particle with energy $e$ in $P_{j}^{-}$, we have

$$
s=j T(e)+\frac{T(e)}{2}-\int_{P_{\beta}-x_{1}}^{x_{0}} \frac{d x}{\sqrt{2(e+\beta(x))}}
$$

if $P_{\beta}-x_{1}<x_{0}$ and

$$
s=j T(e)+\frac{T(e)}{2}+\int_{x_{0}}^{P_{\beta}-x_{1}} \frac{d x}{\sqrt{2(e+\beta(x))}}
$$

if $P_{\beta}-x_{1}>x_{0}$. Above equations of $s$ can be obtained by analyzing the phase space of (2) and here the symmetry property of $\beta((1.5),(1.6))$ is used. By property (IV) of $T(e)$, it is easy to see that right hand sides of (2.15), (2.16), (2.17) and (2.18) are either monotone or convex functions of $e$. So there are at most two particles with different energy such that one of (2.15), (2.16), (2.17) (2.18) is true. Therefore we have $n_{j}^{+}, n_{j}^{-} \leq 2$, for $0 \leq j \leq\left[\frac{s}{T\left(e_{1}\right)}\right]$. So we can use $2\left(\left[\frac{s}{T\left(e_{1}\right)}\right]+1\right)$ intervals $\left[0, P_{\beta}\right]$ to cover the set $\mathbf{X}\left(s ; J_{x}^{+}\right)$. Thus from $(2.14)$,

$$
\int_{J_{x}^{+}}\left|\frac{\partial \mathbf{X}(s)}{\partial v}\right| d v \leq 2\left(\frac{|s|}{T\left(e_{0}\right)}+1\right) P_{\beta} .
$$

Since the estimate for $J_{x}^{-}$is the same, (2.13) is proved. 
Now we can prove the regularity of growing modes.

Proof of Theorem 2.1: Since $f$ defined by (2.1) is differentiable besides the critical set $A_{\text {cr }}$ (defined by (2.4)), almost everywhere we have

$$
\partial_{v} f=-\mu^{\prime \prime}(e) v \phi(x)+\mu^{\prime \prime}(e) v \int_{-\infty}^{0} \lambda e^{\lambda s} \phi(\mathbf{X}(s ; x, v)) d s+I(x, v) .
$$

The first two terms in the right hand side above are obviously integrable. So by Lemma $2.2, \partial_{v} f \in \mathbf{L}^{1}([0, P] \times \mathbf{R})$. Now consider the integrability of $\partial_{x} f$. Besides the set $A_{\mathrm{cr}}, f$ is twice differentiable and satisfy the equation

$$
\lambda f+v \partial_{x} f-E_{0} \partial_{v} f=-\phi_{x} v \mu^{\prime}(e) .
$$

We take $\partial_{x}$ of (2.19) to get

$$
\lambda f_{x}+v \partial_{x}\left(\partial_{x} f\right)-E_{0} \partial_{v}\left(\partial_{x} f\right)=-\beta_{x x} \partial_{v} f-\phi_{x x} v \mu^{\prime}(e)+\phi_{x} \beta_{x} v \mu^{\prime \prime}(e)
$$

From (2.20), we have

$$
\begin{aligned}
\frac{d}{d s}\left(e^{\lambda s} f_{x}(\mathbf{X}(s), \mathbf{V}(s))\right)= & e^{\lambda s}\left(-\beta_{x x} \partial_{v} f-\phi_{x x} v \mu^{\prime}(e)\right. \\
& \left.+\phi_{x} \beta_{x} v \mu^{\prime \prime}(e)(\mathbf{X}(s), \mathbf{V}(s))\right) .
\end{aligned}
$$

Integrating (2.21) from $-\infty$ to 0 , we get

$$
\begin{aligned}
f_{x}(x, v)=\int_{-\infty}^{0} e^{\lambda s}( & -\beta_{x x} \partial_{v} f-\phi_{x x} v \mu^{\prime}(e) \\
& \left.+\phi_{x} \beta_{x} v \mu^{\prime \prime}(e)(\mathbf{X}(s), \mathbf{V}(s))\right) d s .
\end{aligned}
$$


So

$$
\begin{aligned}
& \int_{[0, P]} \int_{\mathbf{R}}\left|f_{x}(x, v)\right| d v d x \\
& \leq \int_{[0, P]} \int_{\mathbf{R}} \int_{-\infty}^{0} e^{\lambda s}\left(\left\|\beta_{x x}\right\|_{\infty}\left|\partial_{v} f\right|+\left|\phi_{x x}\right|\left|v \mu^{\prime}(e)\right|\right. \\
&\left.+\left\|\beta_{x}\right\|_{\infty}\left\|\phi_{x}\right\|_{\infty}\left|v \mu^{\prime \prime}(e)\right|(\mathbf{X}(s), \mathbf{V}(s))\right) d s d v d x \\
&= \int_{-\infty}^{0} e^{\lambda s} \int_{[0, P]} \int_{\mathbf{R}}\left(\left\|\beta_{x x}\right\|_{\infty}\left|\partial_{v} f\right|+\left|\phi_{x x}\right|\left|v \mu^{\prime}(e)\right|\right. \\
&\left.\quad+\left\|\beta_{x}\right\|_{\infty}\left\|\phi_{x}\right\|_{\infty}\left|v \mu^{\prime \prime}(e)\right|(x, v)\right) d v d x d s \\
&=\frac{1}{\lambda} \int_{[0, P]} \int_{\mathbf{R}}\left(\left\|\beta_{x x}\right\|_{\infty}\left|\partial_{v} f\right|+\left|\phi_{x x}\right|\left|v \mu^{\prime}(e)\right|\right. \\
&\left.\quad+\left\|\beta_{x}\right\|_{\infty}\left\|\phi_{x}\right\|_{\infty}\left|v \mu^{\prime \prime}(e)\right|\right) d v d x \\
& \leq \frac{1}{\lambda}\left(\left\|\beta_{x x}\right\|_{\infty}\left\|\partial_{v} f\right\|_{\mathbf{L}^{1}}+\frac{1}{\sqrt{P}} \sup _{x} \int_{\mathbf{R}}\left|v \mu^{\prime}(e)\right| d v\left\|\phi_{x x}\right\|_{2}\right. \\
&\left.\quad+\left\|\beta_{x}\right\|_{\infty}\left\|\phi_{x}\right\|_{\infty}\left\|v \mu^{\prime \prime}(e)\right\|_{\mathbf{L}^{1}}\right) \\
&<\infty . \quad
\end{aligned}
$$

Here in third line, we change the variable

$$
(x, v) \rightarrow(\mathbf{X}(s), \mathbf{V}(s)) .
$$

We shall use the following fact:

Assume a continuous function $a(x, y)$ which is differentiable beyond a closed $\mathbf{C}^{1}$ curve. If $a_{x}, a_{y}$ (defined almost everywhere) are both integrable. Then $a_{x}, a_{y}$ are also the weak derivatives of $a$ in the distribution sense.

It is straightforward to prove the above fact by the definition of weak derivative. Using this fact and the integrability of $f_{x}, f_{v}$ just proved, we know that $f$ defined by $(2.1)$ is in $\mathbf{W}^{1,1}([0, P] \times \mathbf{R})$.

In $([8])$, we showed that $f$ is a weak solution to (2.19). By Theorem $2.1, f$ is also the strong solution to (2.19).

REMARK 2.3 The method of proof of Theorem 2.1 can be used to get the following regularity result: Let $\left(e^{\lambda t} f, e^{\lambda t} E\right)(\operatorname{Re} \lambda>0)$ be a growing mode to (1) with $f \in \mathbf{L}^{1}([0, P] \times \mathbf{R})$, then we have $f \in \mathbf{W}^{1,1}([0, P] \times \mathbf{R})$.

\section{Nonlinear instability of Periodic BGK waves}

By the result in [7], there exists a growing mode to (1). We pick the growing mode $\left(e^{\lambda t} f_{\mathrm{g}}, e^{\lambda t} E_{\mathrm{g}}\right)$ with the largest growth rate $\operatorname{Re} \lambda$. By the 
regularity result proved in the last section, $f_{\mathrm{g}} \in \mathbf{W}^{1,1}([0, P] \times \mathbf{R})$ and $E_{\mathrm{g}} \in \mathbf{W}^{1,1}(0, P)$. Consider the more general case that $\lambda$ is not real. The usual way to construct unstable initial state is to let $\bar{f}^{\delta}(0)=f_{0}+$ $\delta \operatorname{Im} f_{\mathrm{g}}$ and $\bar{E}^{\delta}(0)=E_{0}+\delta \operatorname{Im} E_{\mathrm{g}}$. However to be physically meaningful, the distribution function $f^{\delta}$ must be nonnegative. So some truncation process is required. This was done in [5] for the relativistic case. Here the construction is almost the same as in [5] [6], so we only state the result and indicate some minor modifications.

LEMma 3.1 Let $h(s)$ be either $\langle s\rangle^{\sigma}$ or $\exp (l\langle s\rangle)\left(\langle s\rangle=\sqrt{1+s^{2}}\right)$ with $\sigma \geq 0, l \geq 0$. Assume for sufficiently large $v$, we have

$$
\left|v \mu^{\prime}\left(v^{2} / 2\right)\right| \leq C^{\prime} h(|v|) \mu\left(v^{2} / 2\right), \mu\left(v^{2} / 2\right) \leq h^{\prime}(|v|)[h(|v|)]^{-2-m}
$$

for some $m>0$. Then there exists $\delta_{0}, \theta, c_{0}>0$ such that for $0<\delta<\delta_{0}$, there exists perturbed initial state

$$
\left(\bar{f}^{\delta}(0), \bar{E}^{\delta}(0)\right)=\left(f_{0}+\delta f_{g}^{\delta}, E_{0}+\delta E_{g}^{\delta}\right)
$$

such that

$$
\begin{gathered}
\bar{f}^{\delta}(0) \in \mathbf{W}^{1,1}([0, P] \times \mathbf{R}), \bar{E}^{\delta}(0) \in \mathbf{W}^{1,1}(0, P), \\
\left\|\bar{f}^{\delta}(0)-f_{0}\right\|_{W^{1,1}([0, P] \times \mathbf{R})}+\left\|\bar{E}^{\delta}(0)-E_{0}\right\|_{W^{1,1}(0, P)}=\delta, \\
\bar{f}^{\delta}(0) \geq 0, \\
\partial_{x} E_{g}^{\delta}=-\int_{\mathbf{R}} f_{g}^{\delta} d v .
\end{gathered}
$$

Moreover, denote $\left(f_{L}^{\delta}(t), E_{L}^{\delta}(t)\right)$ be the solution to the linearized equation (1) with $\left(f_{L}^{\delta}(0), E_{L}^{\delta}(0)\right)=\left(\delta f_{g}^{\delta}, \delta E_{g}^{\delta}\right)$, then we have

$$
c_{0} \delta e^{\operatorname{Re} \lambda t} \leq\left\|E_{L}^{\delta}(t)\right\|_{\mathbf{L}^{1}(0, P)} \leq 3 c_{0} \delta e^{\operatorname{Re} \lambda t}
$$

for $0 \leq t \leq T^{*}\left(\right.$ here $\left.\delta e^{\operatorname{Re} \lambda T^{*}}=\theta\right)$.

We can use the same arguments in [5] to prove Lemma 3.1 and we refer to [5] for the details. A minor modification is the following. From (2.1) for the growing mode $f_{\mathrm{g}}$, we have

$$
\left|f_{\mathrm{g}}(x, v)\right| \leq 2\left\|\phi_{\mathrm{g}}\right\|_{\infty}\left|\mu^{\prime}(e)\right| .
$$


This estimate can be used to prove Lemma 14 in [5] without the assumption that $E_{0}$ is small. A minor extension of Lemma 3.1 is to dealt with the case when $f_{0}=\mu(e)$ has compact support. In this case, by assuming the growth condition like (3.1) near the boundary of the support of $\mu$, we can construct corresponding nonnegative $f^{\delta}(0)$ by the similar truncation technique. We skip these details and move on to the nonlinear instability proof below.

Denote $\left(\bar{f}^{\delta}(t), \bar{E}^{\delta}(t)\right)$ to be the solution to (1) with initial data $\left(\bar{f}^{\delta}(0), \bar{E}^{\delta}(0)\right)$ as constructed in Lemma 3.1. Denote

$$
\left(f^{\delta}(t), E^{\delta}(t)\right)=\left(\bar{f}^{\delta}(t)-f_{0}, \bar{E}^{\delta}(t)-E_{0}\right) .
$$

to be the perturbations satisfying the equation

$$
\begin{gathered}
\frac{\partial f}{\partial t}=-v \frac{\partial f}{\partial x}+E_{0} \frac{\partial f}{\partial v}+E \frac{\partial f_{0}}{\partial v}+E \frac{\partial f}{\partial v}, \\
\frac{\partial E}{\partial x}=-\int_{-\infty}^{+\infty} f d v
\end{gathered}
$$

with

$$
\left(f^{\delta}(0), E^{\delta}(0)\right)=\left(\delta f_{\mathrm{g}}^{\delta}, \delta E_{\mathrm{g}}^{\delta}\right)
$$

In the following we consider a fixed $\delta<\delta_{0}$, so we simply denote $\left(\left(\bar{f}^{\delta}(t), \bar{E}^{\delta}(t)\right)\right)$ and $\left(f^{\delta}(t), E^{\delta}(t)\right)$ by $((\bar{f}(t), \bar{E}(t)))$ and $(f(t), E(t))$. Denote

$$
D=-v \cdot \partial_{x}+E_{0} \cdot \partial_{v}
$$

then the linearized operator $L_{0}$ corresponding to the linear part of (3) can be written as

$$
L_{0} f=D f+\mu^{\prime}(e) D \partial_{x x}^{-1}\left(\int_{\mathbf{R}} f d v\right)=D f+K f .
$$

Here we define $\partial_{x x}^{-1}$ in the following way: $\psi=\partial_{x x}^{-1} \rho$ is the solution to

$$
\psi_{x x}=\rho, \text { with } \int_{0}^{P} \psi(x) d x=0 .
$$

Then we have

$$
f(t)=e^{t L_{0}} f(0)+\int_{0}^{t} e^{(t-s) L_{0}}\left(\partial_{v}(E f)(s)\right) d s=f_{L}(t)+f_{N}(t)
$$


and correspondingly

$$
\begin{aligned}
E(t) & =-\partial_{x} \phi(t) \\
& =-\partial_{x} \partial_{x x}^{-1}\left(\int_{\mathbf{R}} f(t) d v\right) \\
& =-\partial_{x} \partial_{x x}^{-1}\left(\int_{\mathbf{R}} f_{L}(t) d v\right)-\partial_{x} \partial_{x x}^{-1}\left(\int_{\mathbf{R}} f_{N}(t) d v\right) \\
& =E_{L}(t)+E_{N}(t)=-\partial_{x} \phi_{L}(t)-\partial_{x} \phi_{N}(t) .
\end{aligned}
$$

We define the dual operator $L_{0}^{*}$ of $L_{0}$ as

$$
L_{0}^{*} f:=-D f-\partial_{x x}^{-1}\left(\int_{\mathbf{R}} \mu^{\prime}(e) D f d v\right)=-D f+K^{*} f .
$$

Consider

$$
e^{t L_{0}}: L^{1}([0, P] \times \mathbf{R}) \rightarrow L^{1}([0, P] \times \mathbf{R})
$$

and

$$
e^{t L_{0}^{*}}: L^{\infty}([0, P] \times \mathbf{R}) \rightarrow L^{\infty}([0, P] \times \mathbf{R})
$$

We have

Lemma 3.2 For any $f \in L^{1}([0, P] \times \mathbf{R}), f^{*} \in L^{\infty}([0, P] \times \mathbf{R})$ and $t \in$ $\mathbf{R}$, the following is true

$$
\iint_{[0, P] \times \mathbf{R}}\left(e^{t L_{0}} f\right) f^{*} d x d v=\iint_{[0, P] \times \mathbf{R}} f\left(e^{t L_{0}^{*}} f^{*}\right) d x d v .
$$

Proof: Since $\mathbf{L}^{1}$ and $\mathbf{L}^{\infty}$ are not dual spaces, we cannot use the abstract theory directly. We sketch the proof below. Let

$$
\begin{gathered}
f_{0}(t, x, v)=e^{t D} f=f(\mathbf{X}(-t ; x, v), \mathbf{V}(-t ; x, v)) \\
f_{1}(t, x, v)=f_{0}+\int_{0}^{t} e^{(t-s) D} K f_{0}(s) d s \\
=f_{0}+\int_{0}^{t}\left(K f_{0}\right)(s, \mathbf{X}(-(t-s) ; x, v), \mathbf{V}(-(t-s) ; x, v)) d s \\
\ldots \\
f_{k+1}(t, x, v)=f_{0}+\int_{0}^{t}\left(K f_{k}\right)(s, \mathbf{X}(-(t-s) ; x, v), \mathbf{V}(-(t-s) ; x, v)) d s
\end{gathered}
$$

and

$$
f_{0}^{*}(t, x, v)=e^{-t D} f^{*}=f^{*}(\mathbf{X}(t ; x, v), \mathbf{V}(t ; x, v))
$$




$$
\begin{gathered}
f_{1}^{*}(t, x, v)=f_{0}^{*}+\int_{0}^{t}\left(K^{*} f_{0}^{*}\right)(s, \mathbf{X}(t-s ; x, v), \mathbf{V}(t-s ; x, v)) d s \\
\ldots \\
f_{k+1}^{*}(t, x, v)=f_{0}^{*}+\int_{0}^{t}\left(K f_{k}^{*}\right)(s, \mathbf{X}(t-s ; x, v), \mathbf{V}(t-s ; x, v)) d s .
\end{gathered}
$$

Then since $K, K^{*}$ are compact operators, by the standard theory we have

$$
f_{k} \rightarrow e^{t L_{0}} f \text { in } L^{1} \text { and } f_{k}^{*} \rightarrow e^{t L_{0}^{*}} f \text { in } L^{\infty}
$$

as $k \rightarrow \infty$. By deduction on $k$ and a tedious computation using integration by parts, we can show that

$$
\iint_{[0, P] \times \mathbf{R}} f_{k} f^{*} d x d v=\iint_{[0, P] \times \mathbf{R}} f f_{k}^{*} d x d v
$$

for any integer $k$. Letting $k \rightarrow \infty$ in the above, we get the conclusion.

We need the following simple lemma.

LEMMA 3.3

(i) If $g(x) \in \mathbf{L}_{p e r}^{1}(0, P)$ and $\int_{0}^{P} g(x) d x=0$, then

$$
\|g\|_{1} \leq 2 \sup _{\substack{a \in \mathbf{W}_{\text {per }}^{1,0, P)} \\\left\|a_{x}\right\|_{\infty} \leq 1}} \int_{0}^{P} g a_{x} d x .
$$

Moreover if $g_{x} \in \mathbf{L}^{1}(0, P)$, then

$$
\|g\|_{1} \leq 2 \sup _{\substack{a \in \mathbf{W}_{\text {per }}^{1, \infty}(0, P) \\\left\|a_{x}\right\|_{\infty} \leq 1}} \int_{0}^{P} g_{x} a d x .
$$

(ii) If $g(x) \in \mathbf{L}_{\text {per }}^{\infty}(0, P)$ and $\int_{0}^{P} g(x) d x=0$, then

$$
\|g\|_{\infty} \leq 2 \sup _{\substack{a \in \mathbf{W}_{p e r}^{1,1}(0, P) \\\left\|a_{x}\right\|_{1} \leq 1}} \int_{0}^{P} g a_{x} d x .
$$

Moreover if $g_{x} \in \mathbf{L}^{\infty}(0, P)$, then

$$
\|g\|_{\infty} \leq 2 \sup _{\substack{a \in \mathbf{W}_{\text {per }}^{1,1}(0, P) \\\left\|a_{x}\right\|_{1} \leq 1}} \int_{0}^{P} g_{x} a d x .
$$


Proof: We only prove (i) since the proof of (ii) is the same. We have

$$
\|g\|_{1}=\sup _{\substack{b \in \mathbf{L}_{\text {per }}^{\infty} \\\|b\|_{\infty}=1}} \int_{0}^{P} g(x) b(x) d x .
$$

Since the function

$$
b-\frac{1}{P} \int_{0}^{P} b(x) d x
$$

has zero integral, there exists some function $a \in \mathbf{W}_{\text {per }}^{1, \infty}(0, P)$ such that

$$
b-\frac{1}{P} \int_{0}^{P} b(x) d x=a_{x} .
$$

So

$$
b=\frac{1}{P} \int_{0}^{P} b(x) d x+a_{x}
$$

and clearly $\left\|a_{x}\right\|_{\infty} \leq 2\|b\|_{\infty}=2$. From (3.8), (3.9) and the assumption that $\int_{0}^{P} g d x=0$, we have

$$
\begin{aligned}
\|g\|_{1} & =\sup _{\substack{b \in \mathbf{L}_{\text {per }}^{\infty} \\
\|b\|_{\infty}=1}} \int_{0}^{P} g(x) a_{x} d x \\
\leq & \sup _{\substack{a \in \mathbf{W}_{\text {per }}^{1, \infty}(0, P) \\
\left\|a_{x}\right\|_{\infty} \leq 2}} \int_{0}^{P} g a_{x} d x .
\end{aligned}
$$

The estimate (3.5) follows from (3.4) by integration by parts.

The following lemma will be used later.

Lemma 3.4 For any $\varepsilon<0$, there exists constant $C_{\varepsilon}$ such that: for any function $a(x)$ in $\mathbf{W}_{\text {per }}^{1, \infty}(0, P)$ with $\left\|a_{x}\right\|_{\infty} \leq 1$, we have the following estimate

$$
\left|\partial_{v}\left(e^{t L_{0}^{*}} a\right)\right| \leq P\left|\frac{\partial \mathbf{X}(t)}{\partial v}\right|+C_{\varepsilon} \int_{0}^{t} e^{(\operatorname{Re} \lambda+\varepsilon) s}\left|\frac{\partial \mathbf{X}(t-s)}{\partial v}\right| d s .
$$

Proof: Denote

$$
h(t, x, v)=e^{t L_{0}^{*}} a,
$$


then $h$ is the solution to the equation

$$
\partial_{t} h=-D h-\partial_{x x}^{-1}\left(\int_{\mathbf{R}} \mu^{\prime}(e) D h d v\right)
$$

with $g(0)=a(x)$. We have the following estimate:

$$
\|h(t)\|_{\infty} \leq C_{\varepsilon}^{\prime} e^{(\operatorname{Re} \lambda+\varepsilon) t}
$$

for some constant $C_{\varepsilon}^{\prime}$. To prove (3.12), we notice that: there exists $C_{\varepsilon}^{\prime \prime}$ such that

$$
\left\|e^{t L_{0}}\right\|_{\mathbf{L}^{1} \rightarrow \mathbf{L}^{1}} \leq C_{\varepsilon}^{\prime \prime} e^{(\operatorname{Re} \lambda+\varepsilon) t} .
$$

This is due to the fact that $L_{0}$ is compact perturbation of the operator $D$ which generate an isometry group in any $L^{p}$ space $(1 \leq p<\infty)$, and $\operatorname{Re} \lambda$ is the maximal growth rate. Now (3.12) follows easily by duality since

$$
\begin{aligned}
\|h(t)\|_{\infty} & =\left\|e^{t L_{0}^{*} a}\right\|_{\infty} \\
& =\sup _{\|k\|_{1}=1} \iint_{[0, P] \times \mathbf{R}}\left(e^{t L_{0}^{*}} a\right) k(x, v) d x d v \\
& =\sup _{\|k\|_{1}=1} \iint_{[0, P] \times \mathbf{R}} a(x)\left(e^{t L_{0}} k\right)(x, v) d x d v \text { (by Lemma 3.2) } \\
& \leq\|a\|_{\infty}\left\|e^{t L_{0}} k\right\|_{1} \leq P\left\|a_{x}\right\|_{1}\left\|e^{t L_{0}}\right\|_{\mathbf{L}^{1} \rightarrow \mathbf{L}^{1}} \\
& \leq P C_{\varepsilon}^{\prime \prime} e^{(\operatorname{Re} \lambda+\varepsilon) t} .
\end{aligned}
$$

From (3.11), we have

$$
\begin{aligned}
h(t) & =e^{-t D} a-\int_{0}^{t} e^{-(t-s) D} \partial_{x x}^{-1}\left(\int_{\mathbf{R}} \mu^{\prime}(e) D h(s) d v\right) d s \\
& =a(\mathbf{X}(t))-\int_{0}^{t} \partial_{x x}^{-1}\left(\int_{\mathbf{R}} \mu^{\prime}(e) D h d v\right)(s, \mathbf{X}(t-s)) d s .
\end{aligned}
$$

So

$$
\begin{aligned}
& \partial_{v} h(t)=a_{x}(\mathbf{X}(t)) \frac{\partial \mathbf{X}(t)}{\partial v} \\
& \quad-\int_{0}^{t} \partial_{x} \partial_{x x}^{-1}\left(\int_{\mathbf{R}} \mu^{\prime}(e) D h d v\right)(s, \mathbf{X}(t-s)) \frac{\partial \mathbf{X}(t-s)}{\partial v} d s .
\end{aligned}
$$

Denoting

$$
g(s, x)=\partial_{x} \partial_{x x}^{-1}\left(\int_{\mathbf{R}} \mu^{\prime}(e) D h(s) d v\right)
$$


then by (3.7) we have

$$
\begin{aligned}
& \|g(s)\|_{\infty} \leq 2 \sup _{\substack{\left.b \in \mathbf{W}_{\text {per }}^{1,0, P}\right) \\
\left\|b_{x}\right\|_{1} \leq 1}} \int_{0}^{P} g_{x} b d x \\
& =2 \sup _{\substack{b \in \mathbf{W}_{\text {per }}^{1,1}(0, P) \\
\left\|b_{x}\right\|_{1} \leq 1}} \iint_{[0, P] \times \mathbf{R}} \mu^{\prime}(e) D h(s) b(x) d x d v \\
& =2 \sup _{\substack{b \in \mathbf{W}_{\text {per }}^{1,1}(0, P) \\
\left\|b_{x}\right\|_{1} \leq 1}} \iint_{[0, P] \times \mathbf{R}} h(s) D\left(\mu^{\prime}(e) b\right) d x d v \\
& =2 \sup _{\substack{b \in \mathbf{W}_{\text {per }}^{1,1}(0, P) \\
\left\|b_{x}\right\|_{1} \leq 1}} \iint_{[0, P] \times \mathbf{R}} h(s) \mu^{\prime}(e) v b_{x} d x d v \\
& \leq 2\|h(s)\|_{\infty} \sup _{x} \int_{\mathbf{R}}\left|v \mu^{\prime}(e)\right| d v\left\|b_{x}\right\|_{1} \\
& \leq 2 C_{\varepsilon}^{\prime} \sup _{x} \int_{\mathbf{R}}\left|v \mu^{\prime}(e)\right| d v e^{(\operatorname{Re} \lambda+\varepsilon) s} \text { (by (3.12)). }
\end{aligned}
$$

Let

$$
C_{\varepsilon}=2 C_{\varepsilon}^{\prime} \sup _{x} \int_{\mathbf{R}}\left|v \mu^{\prime}(e)\right| d v,
$$

then (3.10) follows from (3.13) and the above estimate.

We need the following bootstrap lemma.

Lemma 3.5 For $0<\delta<\delta_{0}$, consider the solution $(E(t), f(t))$ to (3) with

$$
(f(0), E(0))=\left(\delta f_{g}^{\delta}, \delta E_{g}^{\delta}\right) .
$$

If

$$
\|E(t)\|_{\mathbf{L}^{1}(0, P)} \leq 4 c_{0} \delta e^{\operatorname{Re} \lambda t}
$$

for $0 \leq t \leq T^{*}$ (here $\delta_{0}, c_{0}, T^{*}$ are as in Lemma 3.1), then there exists constants $c_{1}, c_{2}, c_{3}$ such that

$$
\|f(t)\|_{1} \leq c_{1} \delta e^{\operatorname{Re} \lambda t},\|f(t)\|_{\infty} \leq c_{2} \delta e^{\operatorname{Re} \lambda t},\|E(t)\|_{\infty} \leq c_{3} \delta e^{\operatorname{Re} \lambda t}
$$

for $0 \leq t \leq T^{*}$. 
Proof: First we estimate $\|f(t)\|_{1}$. We rewrite (3.3a) as

$$
\frac{\partial f}{\partial t}+v \frac{\partial f}{\partial x}-\bar{E}(t) \frac{\partial f}{\partial v}=E \frac{\partial f_{0}}{\partial v}
$$

where $\bar{E}(t)=E_{0}+E(t)$ is the perturbed electric field. Denote

$$
(\overline{\mathbf{X}}(s ; x, v), \overline{\mathbf{V}}(s ; x, v))
$$

to be the solution of the perturbed characteristic equation

$$
\begin{aligned}
\frac{d}{d s} \overline{\mathbf{X}}(s) & =\overline{\mathbf{V}}(s), \\
\frac{d}{d s} \overline{\mathbf{V}}(s) & =-\bar{E}(s, \overline{\mathbf{X}}(s)),
\end{aligned}
$$

with initial data $\overline{\mathbf{X}}(0)=x, \overline{\mathbf{V}}(0)=v$. Then integrating (3.15) along the perturbed trajectory, we have

$$
\begin{aligned}
f(t, x, v) & =f(0, \overline{\mathbf{X}}(-t), \overline{\mathbf{V}}(-t)) \\
& +\int_{0}^{t}\left(E \frac{\partial f_{0}}{\partial v}\right)(s, \overline{\mathbf{X}}(-(t-s)), \overline{\mathbf{V}}(-(t-s))) d s .
\end{aligned}
$$

So

$$
\begin{aligned}
& \iint_{[0, P] \times \mathbf{R}}|f(t, x, v)| d x d v \\
\leq & \iint_{[0, P] \times \mathbf{R}}|f(0, \overline{\mathbf{X}}(-t), \overline{\mathbf{V}}(-t))| d x d v \\
& +\int_{0}^{t} \iint_{[0, P] \times \mathbf{R}}\left|E \frac{\partial f_{0}}{\partial v}\right|(s, \overline{\mathbf{X}}(-(t-s)), \overline{\mathbf{V}}(-(t-s))) d x d v d s \\
= & \iint_{[0, P] \times \mathbf{R}}|f(0)| d x d v+\int_{0}^{t} \iint_{[0, P] \times \mathbf{R}}\left|E \frac{\partial f_{0}}{\partial v}\right|(s) d x d v d s \\
\leq & \delta\left\|f_{\mathrm{g}}^{\delta}\right\|_{1}+\sup _{x} \int_{\mathbf{R}}\left|v \mu^{\prime}(e)\right| d v \int_{0}^{t}\|E(s)\|_{1} d s \\
\leq & \left(\left\|f_{\mathrm{g}}^{\delta}\right\|_{1}+\frac{4 c_{0}}{\operatorname{Re} \lambda} \sup _{x} \int_{\mathbf{R}}\left|v \mu^{\prime}(e)\right| d v\right) \delta e^{\operatorname{Re} \lambda t}:=c_{1} \delta e^{\operatorname{Re} \lambda t} .
\end{aligned}
$$

Here in the second equality above we changed the variable by

$$
(x, v) \rightarrow(\overline{\mathbf{X}}(-(t-s)), \overline{\mathbf{V}}(-(t-s)))
$$


which has Jacobian 1 . The estimate of $\|E(t)\|_{\infty}$ follows easily by letting $c_{3}=P c_{1}$, since

$$
\begin{aligned}
\|E(t)\|_{\infty} & \leq P\left\|\partial_{x} E(t)\right\|_{1} \\
& \leq P\|f(t)\|_{1} \leq P c_{1} \delta e^{\operatorname{Re} \lambda t} .
\end{aligned}
$$

Now we estimate $\|f(t)\|_{\infty}$. Define the electron current

$$
j(t)=\int_{\mathbf{R}} v f(t) d v .
$$

First we need the following estimate

$$
\|j(t)\|_{1} \leq c^{\prime} \delta e^{\operatorname{Re} \lambda t}
$$

for some constant $c^{\prime}$ and $0 \leq t \leq T^{*}$. To show (3.18), we obtain the following equation from (3.15)

$$
\frac{\partial(v f)}{\partial t}+v \frac{\partial(v f)}{\partial x}-\bar{E}(t) \frac{\partial(v f)}{\partial v}=E v \frac{\partial f_{0}}{\partial v}-\bar{E}(t) f .
$$

Integrating above along the perturbed trajectory, we have

$$
\begin{aligned}
v f(t, x, v)=\overline{\mathbf{V}}(-t) f(0, \overline{\mathbf{X}}(-t), \overline{\mathbf{V}}(-t)) \\
\quad+\int_{0}^{t}\left(E v \frac{\partial f_{0}}{\partial v}-\bar{E} f\right)(s, \overline{\mathbf{X}}(-(t-s)), \overline{\mathbf{V}}(-(t-s))) d s .
\end{aligned}
$$

So

$$
\begin{aligned}
& \iint_{[0, P] \times \mathbf{R}}|v f(t)| d x d v \\
\leq & \iint_{[0, P] \times \mathbf{R}}|v f(0)| d x d v \\
& +\int_{0}^{t} \iint_{[0, P] \times \mathbf{R}}\left(\left|E v \frac{\partial f_{0}}{\partial v}\right|+\left(\|E\|_{\infty}+\left\|E_{0}\right\|_{\infty}\right)|f|\right)(s) d x d v .
\end{aligned}
$$

Then by the estimates on $\|f(t)\|_{1}$ and $\|E(t)\|_{\infty}$ just proved, there exists some constant $c^{\prime}$ such that

$$
\|v f(t)\|_{1} \leq c^{\prime} \delta e^{\operatorname{Re} \lambda t}
$$

for $0 \leq t \leq T^{*}$, which implies (3.18). Since

$$
\frac{\partial \bar{f}}{\partial t}+v \frac{\partial \bar{f}}{\partial x}-\bar{E} \frac{\partial \bar{f}}{\partial v}=0
$$


we have

$$
\bar{f}(t, x, v)=\bar{f}(0, \overline{\mathbf{X}}(-t), \overline{\mathbf{V}}(-t))
$$

and thus

$$
\begin{aligned}
f(t) & =\bar{f}(t)-f_{0} \\
& =\mu\left(\frac{1}{2} \overline{\mathbf{V}}(-t)^{2}-\beta(\overline{\mathbf{X}}(-t))\right)-\mu\left(\frac{1}{2} v^{2}-\beta(x)\right)+\delta f_{\mathrm{g}}^{\delta}(\overline{\mathbf{X}}(-t), \overline{\mathbf{V}}(-t)) .
\end{aligned}
$$

If $(\overline{\mathbf{X}}(s ; y, u), \overline{\mathbf{V}}(s ; y, u))$ is a solution to $(3.16)$ with initial data $(y, u)$ then

$$
\frac{d}{d s}\left(\frac{1}{2} \overline{\mathbf{V}}(s)^{2}-\bar{\phi}(s, \overline{\mathbf{X}}(s))\right)=-\bar{\phi}_{s}(s, \overline{\mathbf{X}}(s))
$$

and so

$$
\begin{aligned}
\frac{1}{2} \overline{\mathbf{V}}(t ; y, u)^{2}-\bar{\phi}(t, \overline{\mathbf{X}}(t ; y, u)) & =\frac{1}{2} u^{2}-\bar{\phi}(0, y)-\int_{0}^{t} \bar{\phi}_{s}(s, \overline{\mathbf{X}}(s ; y, u)) d s \\
& =\frac{1}{2} u^{2}-\bar{\phi}(0, y)-\int_{0}^{t} \phi_{s}(s, \overline{\mathbf{X}}(s ; y, u)) d s
\end{aligned}
$$

where $\bar{\phi}(t)=\beta+\phi(t)$ is the perturbed potential. Letting

$$
(y, u)=\overline{\mathbf{X}}(-t ; x, v), \overline{\mathbf{V}}(-t ; x, v),
$$

then we have

$$
\frac{1}{2} \overline{\mathbf{V}}(-t)^{2}-\bar{\phi}(0, \overline{\mathbf{X}}(-t))=\frac{1}{2} v^{2}-\bar{\phi}(t, x)+\int_{0}^{t} \phi_{s}(s, \overline{\mathbf{X}}(s-t ; x, v)) d s .
$$

So

$$
\begin{aligned}
& \frac{1}{2} \overline{\mathbf{V}}(-t)^{2}-\beta(\overline{\mathbf{X}}(-t))-\left(\frac{1}{2} v^{2}-\beta(x)\right) \\
& =\phi(0, \overline{\mathbf{X}}(-t))-\phi(t, x)+\int_{0}^{t} \phi_{s}(s, \overline{\mathbf{X}}(s-t ; x, v)) d s .
\end{aligned}
$$

Combining above we have

$$
\|f(t)\|_{\infty} \leq\left\|\mu^{\prime}\right\|_{\infty}\left(\|\phi(t)\|_{\infty}+\delta\left\|\phi_{\mathrm{g}}^{\delta}\right\|_{\infty}\right)+\int_{0}^{t}\left\|\phi_{s}(s)\right\|_{\infty} d s
$$

Since

$$
\|\phi(t)\|_{\infty} \leq P\|E(t)\|_{1} \leq 4 c_{0} P \delta e^{\operatorname{Re} \lambda t}
$$


and

$$
\begin{aligned}
\left\|\phi_{t}(t)\right\|_{\infty} & \leq P\left\|\partial_{x} \phi_{t}(t)\right\|_{1} \\
& =P\left\|\partial_{t} E(t)\right\|_{1} \leq 2 P\|j(t)\|_{1} \\
& \leq c^{\prime} P \delta e^{\operatorname{Re} \lambda t},
\end{aligned}
$$

by (3.19) there exists $c_{2}$ such that

$$
\|f(t)\|_{\infty} \leq c_{2} \delta e^{\operatorname{Re} \lambda t}
$$

for $0 \leq t \leq T^{*}$. Here we use the estimate

$$
\left\|\partial_{t} E(t)\right\|_{1} \leq 2\|j(t)\|_{1} .
$$

which we prove in the below. Denote the electron density by

$$
\rho(t)=\int_{\mathbf{R}} f(t) d v,
$$

then from (3) we have

$$
\partial_{x} E=-\rho, \partial_{t} \rho=-\partial_{x} j .
$$

So by (3.5) of Lemma 3.3,

$$
\begin{aligned}
& \left\|\partial_{t} E(t)\right\|_{1} \leq 2 \sup _{\substack{a \in \mathbf{W}_{\text {per }}^{1,(0, P)} \\
\left\|a_{x}\right\|_{\infty} \leq 1}} \int_{0}^{P} \partial_{x}\left(\partial_{t} E(t)\right) a(x) d x \\
& =2 \sup _{\substack{a \in \mathbf{W}_{\text {per }}^{1,0}(0, P) \\
\left\|a_{x}\right\|_{\infty} \leq 1}} \int_{0}^{P} \partial_{t} \rho(t) a(x) d x \\
& =2 \sup _{\substack{a \in \mathbf{W}_{\text {per }}^{1, \infty}(0, P) \\
\left\|a_{x}\right\|_{\infty} \leq 1}} \int_{0}^{P} \partial_{x} j(t) a(x) d x \\
& =2 \sup _{\substack{a \in \mathbf{W}_{\text {per }}^{1,0, P)} \\
\left\|a_{x}\right\|_{\infty} \leq 1}} \int_{0}^{P} j(t) a_{x}(x) d x \\
& \leq 2\|j(t)\|_{1} \text {. }
\end{aligned}
$$

This ends the proof of Lemma 3.5.

With the preparations above, we can prove Theorem 1.1 now. 
Proof of Theorem 1.1: Denote $T$ to be the maximal time such that

$$
\|E(t)\|_{1} \leq 4 c_{0} \delta e^{\operatorname{Re} \lambda t} .
$$

Notice that (3.2) implies

$$
\|E(0)\|_{1}=\left\|\delta E_{\mathrm{g}}^{\delta}\right\|_{1} \leq 3 c_{0} \delta
$$

so $T>0$. We claim that $T>T^{*}$, where $T^{*}$ is as in Lemmas 3.1, 3.5. Suppose otherwise, we have $T \leq T^{*}$. By (3.5), we have

$$
\left\|E_{N}(t)\right\|_{1} \leq 2 \sup _{\substack{a \in \mathbf{W}_{\text {per }}^{1, \infty}(0, P) \\\left\|a_{x}\right\|_{\infty} \leq 1}} \int_{0}^{P} \partial_{x} E_{N}(t) a(x) d x .
$$

For any $a \in \mathbf{W}_{\text {per }}^{1, \infty}(0, P)$ with $\left\|a_{x}\right\|_{\infty} \leq 1$, we have

$$
\begin{aligned}
& \int_{0}^{P} \partial_{x} E_{N}(t) a(x) d x \\
= & -\iint_{[0, P] \times \mathbf{R}} f_{N}(t) a(x) d x d v \\
= & -\int_{0}^{t} \iint_{[0, P] \times \mathbf{R}} e^{(t-s) L_{0}}\left(\partial_{v}(E f)(s)\right) a(x) d x d v d s \\
= & -\int_{0}^{t} \iint_{[0, P] \times \mathbf{R}} \partial_{v}(E f)(s)\left(e^{\left.(t-s) L_{0}^{*} a\right) d x d v d s}\right. \\
= & \int_{0}^{t} \iint_{[0, P] \times \mathbf{R}}(E f)(s) \partial_{v}\left(e^{\left.(t-s) L_{0}^{*} a\right)} d x d v d s\right. \\
\leq & \int_{0}^{t} \iint_{[0, P] \times \mathbf{R}}|E f|(s)\left(P\left|\frac{\partial \mathbf{X}(t-s)}{\partial v}\right|\right. \\
= & \left.\int_{0}^{t} \iint_{D_{1} \cup D_{2} \cup D_{5}}(\cdots) d x d v d s+\int_{0}^{t-s} e^{(\operatorname{Re} \lambda+\varepsilon) u}\left|\frac{\partial \mathbf{X}(t-s-u)}{\partial v}\right| d u\right) d x d v d s \\
= & I_{1}(t)+I_{2}(t)+I_{3}(t) .
\end{aligned}
$$

In the above we used Lemma 3.4 with $\varepsilon=\frac{1}{4} \operatorname{Re} \lambda$. Now we estimate $I_{3}(t)$. As showed in Section 2, there exists constant $C_{3}$ such that

$$
\left|\frac{\partial \mathbf{X}(t)}{\partial v}\right| \leq C_{3} e^{\frac{1}{2} \operatorname{Re} \lambda|t|} .
$$


So

$$
\begin{aligned}
I_{1}(t) \leq \int_{0}^{t}\|E(s)\|_{\infty}\|f(s)\|_{1}\left(P C_{3} e^{\frac{1}{2} \operatorname{Re} \lambda(t-s)}\right. \\
\left.\quad+C_{\varepsilon} \int_{0}^{t-s} e^{(\operatorname{Re} \lambda+\varepsilon) u} C_{3} e^{\frac{1}{2} \operatorname{Re} \lambda(t-s-u)} d u\right) d s \\
\leq \int_{0}^{t} c_{1} c_{3}\left(\delta e^{\operatorname{Re} \lambda s}\right)^{2}\left(P C_{3} e^{\frac{1}{2} \operatorname{Re} \lambda(t-s)}\right. \\
\left.\quad+C_{\varepsilon} \int_{0}^{t-s} e^{(\operatorname{Re} \lambda+\varepsilon) u} C_{3} e^{\frac{1}{2} \operatorname{Re} \lambda(t-s-u)} d u\right) d s \\
\leq C_{4}^{\prime}\left(\delta e^{\operatorname{Re} \lambda t}\right)^{2}
\end{aligned}
$$

for some constant $C_{4}^{\prime}$ and $0 \leq t \leq T$. To estimate $I_{2}(t)$, we notice that by $(2.11)$

$$
\begin{aligned}
\iint_{D_{3}}\left|\frac{\partial \mathbf{X}(s)}{\partial v}\right| d x d v & \leq \int_{0}^{P} \int_{J_{x}^{+} \cup J_{x}^{-}}\left|\frac{\partial \mathbf{X}(s)}{\partial v}\right| d v d x \\
& \leq 2\left(\frac{s}{T\left(e_{1}\right)}+1\right) P^{2}
\end{aligned}
$$

Thus

$$
\begin{aligned}
I_{2}(t) \leq \int_{0}^{t}\|E(s)\|_{\infty}\|f(s)\|_{\infty}\left(2 P^{3}\left(\frac{t-s}{T\left(e_{1}\right)}+1\right)\right. \\
\left.\quad+C_{\varepsilon} \int_{0}^{t-s} e^{(\operatorname{Re} \lambda+\varepsilon) u} 2\left(\frac{t-s-u}{T\left(e_{1}\right)}+1\right) P^{2} d u\right) d s \\
\leq \int_{0}^{t} c_{2} c_{3}\left(\delta e^{\operatorname{Re} \lambda s}\right)^{2}\left(2 P^{3}\left(\frac{t-s}{T\left(e_{1}\right)}+1\right)\right. \\
\left.\quad+C_{\varepsilon} \int_{0}^{t-s} e^{(\operatorname{Re} \lambda+\varepsilon) u} 2\left(\frac{t-s-u}{T\left(e_{1}\right)}+1\right) P^{2} d u\right) d s \\
\leq C_{4}^{\prime \prime}\left(\delta e^{\operatorname{Re} \lambda t}\right)^{2}
\end{aligned}
$$

for some constant $C_{4}^{\prime \prime}$ and $0 \leq t \leq T$. The estimate of $I_{3}$ is the same and we have

$$
I_{3}(t) \leq C_{4}^{\prime \prime \prime}\left(\delta e^{\operatorname{Re} \lambda t}\right)^{2}
$$

for some constant $C_{4}^{\prime \prime \prime}$ and $0 \leq t \leq T$. Let $C_{4}=2\left(C_{4}^{\prime}+C_{4}^{\prime \prime}+C_{4}^{\prime \prime \prime}\right)$.

Combining these estimates, we have

$$
\int_{0}^{P} \partial_{x} E_{N}(t) a(x) d x \leq \frac{1}{2} C_{4}\left(\delta e^{\operatorname{Re} \lambda t}\right)^{2}
$$


and by (3.20)

$$
\left\|E_{N}(t)\right\|_{1} \leq C_{4}\left(\delta e^{\operatorname{Re} \lambda t}\right)^{2}
$$

for $0 \leq t \leq T$. So we have

$$
\begin{aligned}
\|E(T)\|_{1} & \leq\left\|E_{L}(T)\right\|_{1}+\left\|E_{N}(T)\right\|_{1} \\
& \leq 3 c_{0} \delta e^{\operatorname{Re} \lambda T}+C_{4}\left(\delta e^{\operatorname{Re} \lambda T}\right)^{2} \\
& \leq\left(3 c_{0}+C_{4} \theta\right)\left(\delta e^{\operatorname{Re} \lambda T}\right) \quad(\text { by }(3.2) \text { and }(3.21)) \\
& <3.5 c_{0} \delta e^{\operatorname{Re} \lambda T},
\end{aligned}
$$

if we choose $\theta$ to be small such that $C_{4} \theta<\frac{1}{2}$. This is a contradiction to the definition of $T$. So we must have $T^{*}<T$. Then

$$
\begin{aligned}
\left\|E\left(T^{*}\right)\right\|_{1} & \geq\left\|E_{L}(T)\right\|_{1}-\left\|E_{N}(T)\right\|_{1} \\
& \geq c_{0} \delta e^{\operatorname{Re} \lambda T^{*}}-C_{4}\left(\delta e^{\operatorname{Re} \lambda T^{*}}\right)^{2}=c_{0} \theta-C_{4} \theta^{2} \\
& >\frac{1}{2} c_{0} \theta
\end{aligned}
$$

if $\theta$ is small such that $C_{4} \theta<\frac{1}{2} c_{0}$. We let $\theta_{0}=\frac{1}{2} c_{0} \theta$ and the proof of Theorem 1.1 is finished.

Acknowledgment. I thanks Walter Strauss and Yan Guo for helpful discussions about this work.

\section{Bibliography}

[1] Bardos, C., Guo, Y., Strauss, W. A. Stable and unstable ideal plane flows. Dedicated to the memory of Jacques-Louis Lions. Chinese Ann. Math. Ser. B 23 (2002), no. 2, 149-164.

[2] Bernstein, I., Greene, J., Kruskal, M., Exact nonlinear plasma oscillations. Phys. Rev. 108 (1957), no. 3, 546-550.

[3] Chafee, N.; Infante, E. F. A bifurcation problem for a nonlinear partial differential equation of parabolic type. Applicable Anal. 4 (1974/75), 17-37.

[4] Guo, Y.; Strauss, W. A. Instability of periodic BGK equilibria. Comm. Pure Appl. Math. 48 (1995), no. 8, 861-894.

[5] Guo, Y.; Strauss, W. A. Relativistic unstable periodic BGK waves. Comput. Appl. Math. 18 (1999), no. 1, 87-122.

[6] Guo, Y.; Strauss, W. A. Unstable BGK solitary waves and collisionless shocks. Comm. Math. Phys. 195 (1998), no. 2, 267-293. 
[7] Lin, Z. Instability of periodic BGK waves. Math. Res. Lett. 8 (2001), no. 4, 521534 .

[8] Lin, Z. Stability and instability of equilibria in collisionless plasmas and ideal plane flows, Doctoral dissertation, Brown University, 2003.

[9] Lin, Z. Nonlinear instability of ideal plane flows. Preprint, 2003.

Received Month 199X. 